\title{
Additive resonances of a controlled van der Pol-Duffing oscillator
}

This paper has been published in Journal of Sound and Vibration, vol. 315, issue 1-2, 2008, pp.22-33.

J.C. Ji， N. Zhang

Faculty of Engineering

University of Technology, Sydney

PO Box 123, Broadway, NSW 2007, Australia

Email: jin.ji@uts.edu.au

Total number of pages: 29

Total number of figures: 3 


\title{
Additive resonances of a controlled van der Pol-Duffing oscillator
}

\begin{abstract}
The trivial equilibrium of a controlled van der Pol-Duffing oscillator with nonlinear feedback control may lose its stability via a non-resonant interaction of two Hopf bifurcations when two critical time delays corresponding to two Hopf bifurcations have the same value. Such an interaction results in a non-resonant bifurcation of codimension two. In the vicinity of the non-resonant Hopf bifurcations, the presence of a periodic excitation in the controlled oscillator can induce three types of additive resonances in the forced response, when the frequency of the external excitation and the frequencies of the two Hopf bifurcations satisfy a certain relationship.
\end{abstract}

With the aid of centre manifold theorem and the method of multiple scales, three types of additive resonance responses of the controlled system are investigated by studying the possible solutions and their stability of the four-dimensional ordinary differential equations on the centre manifold. The amplitudes of the free-oscillation terms are found to admit three solutions; two non-trivial solutions and the trivial solution. Of two nontrivial solutions one is stable and the trivial solution is unstable. A stable non-trivial solution corresponds to a quasi-periodic motion of the original system. It is also found that the frequency response curves for three cases of additive resonances are an isolated closed curve. It is shown that the forced response of the oscillator may exhibit quasiperiodic motions on a three-dimensional torus consisting of three frequencies; the frequencies of two bifurcating solutions and the frequency of the excitation. Illustrative examples are given to show the quasi-periodic motions. 


\section{Introduction}

In the absence of external excitation, the equilibrium of a controlled nonlinear system involving time delay may lose its stability and give rise to periodic solutions via Hopf bifurcations, when the time delay reaches certain values. In the neighbourhood of the critical point of Hopf bifurcations, an interaction of bifurcating periodic solutions and an external excitation may induce rich dynamic behaviour. The forced behaviour of the non-autonomous system may exhibit non-resonant response, primary resonances, sub-harmonic and super-harmonic resonances, depending on the relationship of the frequency of Hopf bifurcation and the forcing frequency. The effect of time delays on the stability and dynamics of time delayed systems have received considerable interest in the literature [1-8]. However, there has been less effort made in studying an interaction of the external excitation and two bifurcating solutions which result from non-resonant Hopf bifurcations of the corresponding autonomous systems. The main purpose of the present paper is to study an interaction of the forcing and bifurcating periodic solutions in the vicinity of non-resonant bifurcation of co-dimension two, which appears after the trivial solution of the van der Pol-Duffing oscillator loses its stability via non-resonant Hopf bifurcations.

An externally forced van der Pol-Duffing oscillator under a linear-plus-nonlinear feedback control considered in the present paper is of the form

$$
\begin{aligned}
& \ddot{x}-\left(\mu-\beta x^{2}\right) \dot{x}+\omega^{2} x+\alpha x^{3}=e_{0} \cos \left(\Omega_{0} t\right)+p x(t-\tau)+q \dot{x}(t-\tau)+k_{1} x^{3}(t-\tau) \\
& \quad+k_{2} \dot{x}^{3}(t-\tau)+k_{3} \dot{x}(t-\tau) x^{2}(t-\tau)+k_{4} \dot{x}^{2}(t-\tau) x(t-\tau)
\end{aligned}
$$

where $x$ is the displacement, an overdot indicates the differentiation with respect to time $t, \omega$ is the natural frequency, $\alpha$ is the coefficient of the nonlinear term, $\mu$ and $\beta$ are the linear and nonlinear damping coefficients with $\mu>0, \beta>0, e_{0}$ and $\Omega_{0}$ represent 
the amplitude and frequency of the external excitation, $p$ and $q$ are the proportional and derivative linear feedback gains of a linear-plus-nonlinear feedback control scheme, $k_{i}$ $(i=1,2,3,4)$ are the weakly nonlinear feedback gains, and $\tau$ denotes the time delay occurring in the feedback path. Only one time delay is considered here for simplicity.

The corresponding autonomous system for which the external excitation is neglected in equation (1), which can be obtained by letting $e_{0}=0$ in equation (1), is given by

$$
\begin{gathered}
\ddot{x}-\mu \dot{x}+\omega^{2} x-p x(t-\tau)-q \dot{x}(t-\tau)+\beta x^{2} \dot{x}+\alpha x^{3}-k_{1} x^{3}(t-\tau)-k_{2} \dot{x}^{3}(t-\tau) \\
-k_{3} \dot{x}(t-\tau) x^{2}(t-\tau)-k_{4} \dot{x}^{2}(t-\tau) x(t-\tau)=0
\end{gathered}
$$

It was shown that the trivial equilibrium of the autonomous system (2) may lose its stability via a subcritical or a supercritical Hopf bifurcation and regain its stability via a reverse subcritical or a supercritical Hopf bifurcation as the time delay increases [9]. It was found that an interaction of two Hopf bifurcations may occur when the two critical time delays corresponding to two Hopf bifurcations have the same value. In the vicinity of non-resonant Hopf bifurcations, the controlled oscillator modelled by equation (2) was found to have the initial equilibrium solution, two periodic solutions and a quasiperiodic solution on a two-dimensional (2D) torus [10].

The presence of an external periodic excitation can induce complicated dynamic behaviour of the controlled oscillator given by equation (1), which includes two types of primary resonances, two types of sub-harmonic resonances, two types of superharmonic resonances, three types of additive resonances, and four types of difference resonances [11]. These resonances result from an interaction of the external excitation and the bifurcating periodic solutions that immediately follow the non-resonant Hopf bifurcations of codimension two occurring in the corresponding autonomous system given by equation (2). 
By following the normal procedure for the reduction of delay differential equations to ordinary differential equations based on semigroups of transformations and the decomposition theory [12-14], the dynamic behaviour of the solutions of equation (1) in the neighbourhood of non-resonant Hopf-Hopf interactions can be interpreted by the solutions and their stability of a set of four ordinary differential equations on the centre manifolds.

For simplicity, it is assumed that an intersection of non-resonant Hopf bifurcations occurs at the point $\left(p_{0}, q_{0}, \tau_{0}\right)$, where the corresponding characteristic equation of the autonomous system (2) has two pairs of purely imaginary roots $\pm \mathrm{i} \delta_{01}, \pm \mathrm{i} \delta_{02}$, and all other roots have negative real parts. In order to study the dynamics of the controlled oscillator in the neighbourhood of the bifurcation point $\left(p_{0}, q_{0}, \tau_{0}\right)$, three small perturbation parameters, namely $\alpha_{1}, \alpha_{2}$ and $\alpha_{3}$, are introduced in equation (1) in terms of $\quad p=p_{0}+\alpha_{1}, \quad q=q_{0}+\alpha_{2}, \quad \tau=\tau_{0}+\alpha_{3}$. These perturbation parameters can conveniently account for the small variations of the critical linear feedback gains and the critical time delay.

By treating the external excitation in equation (1) as an additional perturbation term and performing similar algebraic manipulations to those done in reference [10], the four-dimensional ordinary differential equations governing the local flow on the centre manifold can be expressed in the component form as

$$
\begin{aligned}
& \dot{z}_{1}=l_{11} z_{1}+\left(\delta_{1}+l_{12}\right) z_{2}+l_{13} z_{3}+l_{14} z_{4}+f_{10}\left(z_{1}, z_{2}, z_{3}, z_{4}\right)+e_{10} \cos (\Omega t), \\
& \dot{z}_{2}=\left(-\delta_{1}+l_{21}\right) z_{1}+l_{22} z_{2}+l_{23} z_{3}+l_{24} z_{4}+f_{20}\left(z_{1}, z_{2}, z_{3}, z_{4}\right)+e_{20} \cos (\Omega t), \\
& \dot{z}_{3}=l_{31} z_{1}+l_{32} z_{2}+l_{33} z_{3}+\left(\delta_{2}+l_{34}\right) z_{4}+f_{30}\left(z_{1}, z_{2}, z_{3}, z_{4}\right)+e_{30} \cos (\Omega t), \\
& \dot{z}_{4}=l_{41} z_{1}+l_{42} z_{2}+\left(-\delta_{2}+l_{43}\right) z_{3}+l_{44} z_{4}+f_{40}\left(z_{1}, z_{2}, z_{3}, z_{4}\right)+e_{40} \cos (\Omega t),
\end{aligned}
$$


where $\delta_{1}$ and $\delta_{2}$ are the normalized frequencies of Hopf bifurcations which have been rescaled in the units of the critical time delay $\tau_{0}, e_{10}=b_{12} e_{0}, e_{20}=b_{22} e_{0}, e_{30}=b_{32} e_{0}$, $e_{40}=b_{42} e_{0}$, the other coefficients and polynomial functions of order three $f_{i 0}\left(z_{1}, z_{2}, z_{3}, z_{4}\right)$ (where $\left.i=1,2,3,4\right)$ involved in equation (3) are explicitly given in Section 3 of reference [10].

Depending on the relationship of the two natural frequencies $\delta_{1}$ and $\delta_{2}$ with the forcing frequency $\Omega$, the nonlinear system given by equation (3) may exhibit either non-resonant or resonant response. Primary, sub-harmonic and super-harmonic resonances, additive and difference resonances may occur in the forced response. The non-resonant response and primary resonance response of the system have been studied in reference [11] using the method of multiple scales [15]. It was shown that the nonresonant response of the forced oscillator may exhibit quasi-periodic motions on a $2 \mathrm{D}$ or 3D torus. The resonant response may exhibit either periodic motion or quasi-periodic motions on a $2 \mathrm{D}$ torus. The present paper focuses on studying three types of additive resonances, when the forcing frequency is nearly equal to half the sum of the first and second natural frequencies (i.e., $\left.\Omega \cong\left(\delta_{1}+\delta_{2}\right) / 2\right)$; or the sum of the first frequency and twice the second frequency (i.e., $\Omega \cong \delta_{1}+2 \delta_{2}$ ); or the sum of the second frequency and twice the first frequency (i.e., $\Omega \cong 2 \delta_{1}+\delta_{2}$ ).

A closed form of solutions to equation (3) cannot be found analytically. The approximate solutions to additive resonance response of equation (3) will be obtained using the method of multiple scales. The dynamic behaviour of the controlled system in the neighbourhood of the point of non-resonant bifurcations of codimension two will be explored by studying the solutions of a set of four averaged equations that determine the amplitudes and phases of the free oscillation terms in additive resonance response. 
It is assumed that the approximate solutions to equation (3) in the neighbourhood of the trivial equilibrium are represented by an expansion of the form

$$
Z_{i}(t ; \varepsilon)=\varepsilon^{\frac{1}{2}} Z_{i 1}\left(T_{0}, T_{1}, \cdots\right)+\varepsilon^{\frac{3}{2}} Z_{i 2}\left(T_{0}, T_{1}, \cdots\right)+\cdots,(i=1,2,3,4) .
$$

where $\varepsilon$ is a non-dimensional small parameter, and the new multiple independent variables of time are introduced according to $T_{k}=\varepsilon^{k} t, k=0,1,2, \cdots$.

Substituting the approximate solutions (4) into equation (3) and then balancing the like powers of $\varepsilon$ results in the following ordered perturbation equations

$\varepsilon^{\frac{1}{2}}: \quad D_{0} z_{11}=\delta_{1} z_{21}+e_{1} \cos \left(\Omega T_{0}\right)$,

$$
\begin{gathered}
D_{0} z_{21}=-\delta_{1} z_{11}+e_{2} \cos \left(\Omega T_{0}\right), \\
D_{0} z_{31}=\delta_{2} z_{41}+e_{3} \cos \left(\Omega T_{0}\right), \\
D_{0} z_{41}=-\delta_{2} z_{31}+e_{4} \cos \left(\Omega T_{0}\right) .
\end{gathered}
$$

$\varepsilon^{\frac{3}{2}}: \quad D_{0} z_{12}=g_{11}\left(z_{j 1}\right)+\delta_{1} z_{22}-D_{1} z_{11}+f_{11}\left(z_{j 1}\right)$,

$$
\begin{aligned}
& D_{0} z_{22}=g_{21}\left(z_{j 1}\right)-\delta_{1} z_{12}-D_{1} z_{21}+f_{21}\left(z_{j 1}\right), \\
& D_{0} z_{32}=g_{31}\left(z_{j 1}\right)+\delta_{2} z_{42}-D_{1} z_{31}+f_{31}\left(z_{j 1}\right), \\
& D_{0} z_{42}=g_{41}\left(z_{j 1}\right)-\delta_{2} z_{32}-D_{1} z_{41}+f_{41}\left(z_{j 1}\right),
\end{aligned}
$$

where $D_{0}=\partial / \partial T_{0}, D_{1}=\partial / \partial T_{1}$, the coefficients of the perturbation linear terms $l_{i j}$ in equation (3) have been rescaled in terms of $l_{i j}=\varepsilon \bar{l}_{i j}$ and the overbars in $\bar{l}_{i j}$ have been removed for brevity. The $g_{i 1}\left(z_{j 1}\right) \quad(i=1,2,3,4)$ are linear functions of $z_{j 1}$ $(j=1,2,3,4)$ which are given by

$$
\begin{array}{ll}
g_{11}\left(z_{j 1}\right)=l_{11} z_{11}+l_{12} z_{21}+l_{13} z_{31}+l_{14} z_{41}, & g_{21}\left(z_{j 1}\right)=l_{21} z_{11}+l_{22} z_{21}+l_{23} z_{31}+l_{24} z_{41}, \\
g_{31}\left(z_{j 1}\right)=l_{31} z_{11}+l_{32} z_{21}+l_{33} z_{31}+l_{34} z_{41}, & g_{41}\left(z_{j 1}\right)=l_{41} z_{11}+l_{42} z_{21}+l_{43} z_{31}+l_{44} z_{41} .
\end{array}
$$


The $f_{i 1}\left(z_{j 1}\right)$ denotes nonlinear functions of $z_{j 1}(j=1,2,3,4)$ which have been solved from equations (5) and the amplitudes of the excitations in equation (3) have been rescaled in terms of $e_{10}=\varepsilon^{\frac{1}{2}} e_{1}, e_{20}=\varepsilon^{\frac{1}{2}} e_{2}, e_{30}=\varepsilon^{\frac{1}{2}} e_{3}, e_{40}=\varepsilon^{\frac{1}{2}} e_{4}$.

The solutions to equation (5) can be written in a general form as

$$
\begin{aligned}
& z_{11}=r_{1} \cos \left(\delta_{1} T_{0}+\phi_{1}\right)+A_{1} \cos \left(\Omega T_{0}\right)+A_{2} \sin \left(\Omega T_{0}\right), \\
& z_{21}=-r_{1} \sin \left(\delta_{1} T_{0}+\phi_{1}\right)+B_{1} \cos \left(\Omega T_{0}\right)+B_{2} \sin \left(\Omega T_{0}\right), \\
& z_{31}=r_{2} \cos \left(\delta_{2} T_{0}+\phi_{2}\right)+A_{3} \cos \left(\Omega T_{0}\right)+A_{4} \sin \left(\Omega T_{0}\right), \\
& z_{41}=-r_{2} \sin \left(\delta_{2} T_{0}+\phi_{2}\right)+B_{3} \cos \left(\Omega T_{0}\right)+B_{4} \sin \left(\Omega T_{0}\right),
\end{aligned}
$$

where $r_{1}, r_{2}, \phi_{1}, \phi_{2}$ represent, respectively, the amplitudes and phases of the freeoscillation terms, and the coefficients of the particular solutions are given by

$$
\begin{aligned}
& A_{1}=\delta_{1} e_{2} /\left(\delta_{1}^{2}-\Omega^{2}\right), A_{2}=-\Omega e_{1} /\left(\delta_{1}^{2}-\Omega^{2}\right), B_{1}=\left(\Omega A_{2}-e\right) / \delta_{1}, B_{2}=-\Omega A_{1} / \delta_{1}, \\
& A_{3}=\delta_{2} e_{4} /\left(\delta_{2}^{2}-\Omega^{2}\right), A_{4}=-\Omega e_{3} /\left(\delta_{2}^{2}-\Omega^{2}\right), B_{3}=\left(\Omega A_{4}-e_{3}\right) / \delta_{2}, B_{4}=-\Omega A_{3} / \delta_{2} .
\end{aligned}
$$

Differentiating the first and third equation of equation (6) and then substituting the second and fourth equation into the resultant equations results in

$$
\begin{aligned}
& D_{0}^{2} z_{12}+\delta_{1}^{2} z_{12}=D_{0} g_{11}\left(z_{j 1}\right)-D_{0} D_{1} z_{11}+D_{0} f_{11}\left(z_{j 1}\right)+\delta_{1} g_{21}\left(z_{j 1}\right)-\delta_{1} D_{1} z_{21}+\delta_{1} f_{21}\left(z_{j 1}\right), \\
& D_{0}^{2} z_{32}+\delta_{2}^{2} z_{32}=D_{0} g_{31}\left(z_{j 1}\right)-D_{0} D_{1} z_{31}+D_{0} f_{31}\left(z_{j 1}\right)+\delta_{2} g_{41}\left(z_{j 1}\right)-\delta_{2} D_{1} z_{41}+\delta_{2} f_{41}\left(z_{j 1}\right) .
\end{aligned}
$$

Substituting solutions given by equation (7) into the right hand sides of equation (8) yields 44 terms involving trigonometric functions, some of which may produce secular or nearly secular terms in seeking the second-order solutions; $z_{12}$ and $z_{32}$. In addition to four secular terms that are proportional to $\sin \left(\delta_{1} t+\phi_{1}\right), \cos \left(\delta_{1} t+\phi_{1}\right), \sin \left(\delta_{2} t+\phi_{2}\right)$, and $\cos \left(\delta_{2} t+\phi_{2}\right)$, nearly secular terms for additive resonances may appear when 
$\Omega \cong\left(\delta_{1}+\delta_{2}\right) / 2$, or $\Omega \cong 2 \delta_{1}+\delta_{2}$, or $\Omega \cong \delta_{1}+2 \delta_{2}$. These three cases of additive resonances will be referred to here as Cases I, II and III, respectively.

The remainder of the present paper proceeds as follows. In the next section, three types of additive resonance responses of the controlled system are analytically studied using the method of multiple scales. In Section 3, illustrative examples are given to show the frequency-response curves and time histories of additive resonance response of the controlled system. Conclusion is given in Section 4.

\section{Additive Resonances}

To account for the nearness of the forcing frequency to the combination of two natural frequencies for three types of additive resonances, three detuning parameters, namely $\sigma_{1}, \sigma_{2}$ and $\sigma_{3}$, are introduced as follows:

$$
\begin{aligned}
& 2 \Omega=\delta_{1}+\delta_{2}+\varepsilon \sigma_{1}, \\
& \Omega=\delta_{1}+2 \delta_{2}+\varepsilon \sigma_{2}, \\
& \Omega=2 \delta_{1}+\delta_{2}+\varepsilon \sigma_{3} .
\end{aligned}
$$

The averaged equations of the amplitudes and phases for three types of additive resonances will be subsequently obtained using the method of multiple scales.

Case I: $2 \Omega=\delta_{1}+\delta_{2}+\varepsilon \sigma_{1}$

In seeking the second-order solutions for additive resonance Case I from equation (8), the secular terms are the terms of trigonometric functions having the arguments $\left(\delta_{1} t+\phi_{1}\right)$ and $\left(\delta_{2} t+\phi_{2}\right)$, and the nearly secular terms are the trigonometric terms with the arguments $\left(2 \Omega t-\delta_{2} t-\phi_{2}\right)$ and $\left(2 \Omega t-\delta_{1} t-\phi_{1}\right)$. 
Elimination of these secular or nearly secular terms gives rise to the following averaged equations that determine the amplitudes and phases of the free-oscillation terms in equation (7):

$$
\begin{aligned}
& \dot{r}_{1}=-\mu_{1} r_{1}+s_{11} r_{1}^{3}+s_{12} r_{1} r_{2}^{2}+s_{13} r_{2} \cos \left(\gamma_{11}+\gamma_{12}\right)-s_{33} r_{2} \sin \left(\gamma_{11}+\gamma_{12}\right), \\
& r_{1} \dot{\gamma}_{11}=\left(\frac{1}{2} \sigma_{1}-\rho_{1}\right) r_{1}-s_{31} r_{1}^{3}-s_{32} r_{1} r_{2}^{2}-s_{13} r_{2} \sin \left(\gamma_{11}+\gamma_{12}\right)-s_{33} r_{2} \cos \left(\gamma_{11}+\gamma_{12}\right), \\
& \dot{r}_{2}=-\mu_{2} r_{2}+s_{21} r_{1}^{2} r_{2}+s_{22} r_{2}^{3}+s_{23} r_{1} \cos \left(\gamma_{11}+\gamma_{12}\right)-s_{43} r_{1} \sin \left(\gamma_{11}+\gamma_{12}\right), \\
& r_{2} \dot{\gamma}_{12}=\left(\frac{1}{2} \sigma_{1}-\rho_{2}\right) r_{2}-s_{41} r_{1}^{2} r_{2}-s_{42} r_{2}^{3}-s_{23} r_{1} \sin \left(\gamma_{11}+\gamma_{12}\right)-s_{43} r_{1} \cos \left(\gamma_{11}+\gamma_{12}\right),
\end{aligned}
$$

where $\gamma_{11}=\frac{1}{2} \sigma_{1} T_{0}-\phi_{11}, \gamma_{12}=\frac{1}{2} \sigma_{1} T_{0}-\phi_{12}$,

$$
\begin{aligned}
& s_{13}=\frac{1}{8 \delta_{1}}\left(4 \Omega a_{133} A_{1} A_{3} b_{12}-2 \Omega a_{134} A_{2} A_{3} b_{12}+6 \Omega a_{333} A_{3}^{2} b_{12}+\cdots\right), \\
& S_{23}=\frac{1}{8 \delta_{2}}\left(4 \Omega a_{113} A_{1} A_{3} b_{32}-2 \Omega a_{123} A_{2} A_{3} b_{32}+2 \Omega a_{133} A_{3}^{2} b_{32}+\cdots\right), \\
& S_{33}=\frac{1}{8 \delta_{1}}\left(2 \Omega a_{134} A_{1} A_{2} b_{12}+4 \Omega a_{133} A_{2} A_{3} b_{12}+2 \Omega a_{334} A_{3}^{2} b_{12}+\cdots\right), \\
& S_{43}=\frac{1}{8 \delta_{2}}\left(-2 \Omega a_{123} A_{1} A_{3} b_{32}-4 \Omega a_{113} A_{2} A_{3} b_{32}-2 \Omega a_{233} A_{3}^{2} b_{32}+\cdots\right),
\end{aligned}
$$

and the other coefficients, namely $\mu_{1}, \rho_{1}, s_{11}, s_{12}, s_{31}, s_{32}, \mu_{2}, \rho_{2}, s_{21}, s_{22}, s_{41}, s_{42}$ have the same expressions as those obtained for non-resonant response in Section 4 of reference [11]. For the sake of brevity, they are not reproduced in the present paper.

Elimination of the trigonometric terms in equation (12) gives rise to the so-called frequency-response equations:

$$
\begin{aligned}
& r_{1}^{2}\left(-\mu_{1}+s_{11} r_{1}^{2}+s_{12} r_{2}^{2}\right)^{2}+r_{1}^{2}\left(\frac{1}{2} \sigma_{1}-\rho_{1}-s_{31} r_{1}^{2}-s_{32} r_{2}^{2}\right)^{2}-\left(s_{13}^{2}+s_{33}^{2}\right) r_{2}^{2}=0, \\
& r_{2}^{2}\left(-\mu_{2}+s_{21} r_{1}^{2}+s_{22} r_{2}^{2}\right)^{2}+r_{2}^{2}\left(\frac{1}{2} \sigma_{1}-\rho_{2}-s_{41} r_{1}^{2}-s_{42} r_{2}^{2}\right)^{2}-\left(s_{23}^{2}+s_{43}^{2}\right) r_{1}^{2}=0 .
\end{aligned}
$$

The coefficients in equation (13) can be numerically obtained for a specific system with a given set of system parameters. Then equation (13) can be numerically solved using 
the Newton-Raphson procedure. The stability of the steady state solutions to equation (13) can be examined by computing the eigenvalues of the coefficient matrix of characteristic equations, which are derived from equation (12) in terms of small disturbances to the steady state solutions.

As the averaged equation (12) involves the coupled terms $r_{1} \dot{\gamma}_{11}$ and $r_{2} \dot{\gamma}_{12}$, the perturbation equations will not contain the perturbations $\Delta \dot{\gamma}_{11}$ and $\Delta \dot{\gamma}_{12}$ for the trivial solution and hence the stability of the trivial solution cannot be studied by directly perturbing equation (12). To overcome this difficulty, normalization method is used by introducing the transformation $p_{11}=r_{1} \cos \gamma_{11}, \quad q_{11}=r_{1} \sin \gamma_{11}, \quad p_{12}=r_{2} \cos \gamma_{12}$, $q_{12}=r_{2} \sin \gamma_{12}$, into equation (12). Performing trigonometric manipulations leads to the following modulation equations in the Cartesian form:

$$
\begin{aligned}
\dot{p}_{11}= & -\mu_{1} p_{11}+\left(\rho_{1}-\frac{1}{2} \sigma_{1}\right) q_{11}+s_{13} p_{12}-s_{33} q_{12}+s_{11} p_{11}\left(p_{11}^{2}+q_{11}^{2}\right)+s_{12} p_{11}\left(p_{12}^{2}+q_{12}^{2}\right) \\
& +s_{31} q_{11}\left(p_{11}^{2}+q_{11}^{2}\right)+s_{32} q_{11}\left(p_{12}^{2}+q_{12}^{2}\right), \\
\dot{q}_{11}= & -\mu_{1} q_{11}-\left(\rho_{1}-\frac{1}{2} \sigma_{1}\right) p_{11}-s_{33} p_{12}-s_{13} q_{12}+s_{11} q_{11}\left(p_{11}^{2}+q_{11}^{2}\right)+s_{12} q_{11}\left(p_{12}^{2}+q_{12}^{2}\right) \\
& -s_{31} p_{11}\left(p_{11}^{2}+q_{11}^{2}\right)-s_{32} p_{11}\left(p_{12}^{2}+q_{12}^{2}\right), \\
\dot{p}_{12}= & -\mu_{2} p_{12}+\left(\rho_{2}-\frac{1}{2} \sigma_{1}\right) q_{12}+s_{23} p_{11}-s_{43} q_{11}+s_{21} p_{12}\left(p_{11}^{2}+q_{11}^{2}\right)+s_{22} p_{12}\left(p_{12}^{2}+q_{12}^{2}\right) \\
& +s_{41} q_{12}\left(p_{11}^{2}+q_{11}^{2}\right)+s_{42} q_{12}\left(p_{12}^{2}+q_{12}^{2}\right), \\
\dot{q}_{12}= & -\mu_{2} q_{12}-\left(\rho_{2}-\frac{1}{2} \sigma_{1}\right) p_{12}-s_{43} p_{11}-s_{23} q_{11}+s_{21} q_{12}\left(p_{11}^{2}+q_{11}^{2}\right)+s_{22} q_{12}\left(p_{12}^{2}+q_{12}^{2}\right) \\
& -s_{41} p_{12}\left(p_{11}^{2}+q_{11}^{2}\right)-s_{42} p_{12}\left(p_{12}^{2}+q_{12}^{2}\right) .
\end{aligned}
$$

The stability of the steady state solutions is determined by the eigenvalues of the corresponding Jacobian matrix of equation (14). The resultant characteristic equations for both trivial and non-trivial solutions depend in a complicated manner on the system and forcing parameters. Specific results are therefore at best obtained numerically. 
Case II: $\Omega=\delta_{1}+2 \delta_{2}+\varepsilon \sigma_{2}$

Secular and nearly secular terms for additive resonance Case II are terms that are proportional to the trigonometric terms with the arguments of $\left(\delta_{1} t+\phi_{1}\right),\left(\delta_{2} t+\phi_{2}\right)$, $\left(\Omega t-2 \delta_{2} t-2 \phi_{2}\right)$ and $\left(\Omega t-\delta_{1} t-\delta_{2} t-\phi_{1}-\phi_{2}\right)$. Eliminating these secular and nearly secular terms in seeking the second-order solutions yields the averaged equations for additive resonance Case II:

$$
\begin{aligned}
& \dot{r}_{1}=-\mu_{1} r_{1}+s_{11} r_{1}^{3}+s_{12} r_{1} r_{2}^{2}+s_{14} r_{2}^{2} \cos \left(\gamma_{21}+2 \gamma_{22}\right)-s_{34} r_{2}^{2} \sin \left(\gamma_{21}+2 \gamma_{22}\right), \\
& r_{1} \dot{\gamma}_{21}=\left(\frac{1}{3} \sigma_{2}-\rho_{1}\right) r_{1}-s_{31} r_{1}^{3}-s_{32} r_{1} r_{2}^{2}-s_{14} r_{2}^{2} \sin \left(\gamma_{21}+2 \gamma_{22}\right)-s_{34} r_{2}^{2} \cos \left(\gamma_{21}+2 \gamma_{22}\right), \\
& \dot{r}_{2}=-\mu_{2} r_{2}+s_{21} r_{1}^{2} r_{2}+s_{22} r_{2}^{3}+s_{24} r_{1} r_{2} \cos \left(\gamma_{21}+2 \gamma_{22}\right)-s_{44} r_{1} r_{2} \sin \left(\gamma_{21}+2 \gamma_{22}\right), \\
& r_{2} \dot{\gamma}_{22}=\left(\frac{1}{3} \sigma_{2}-\rho_{2}\right) r_{2}-s_{41} r_{1}^{2} r_{2}-s_{42} r_{2}^{3}-s_{24} r_{1} r_{2} \sin \left(\gamma_{21}+2 \gamma_{22}\right)-s_{44} r_{1} r_{2} \cos \left(\gamma_{21}+2 \gamma_{22}\right),
\end{aligned}
$$

where $\gamma_{21}=\frac{1}{3} \sigma_{2} T_{0}-\phi_{21}, \gamma_{22}=\frac{1}{3} \sigma_{2} T_{0}-\phi_{22}$,

$$
\begin{aligned}
& s_{14}=\frac{1}{8 \delta_{1}}\left(-\Omega a_{134} A_{2} b_{12}+3 \Omega a_{333} A_{3} b_{12}-\Omega a_{344} A_{3} b_{12}+\cdots\right), \\
& s_{24}=\frac{1}{8 \delta_{2}}\left(2 \Omega a_{133} A_{3} b_{32}-2 \Omega a_{114} A_{2} b_{32}-\Omega a_{123} A_{2} b_{32}+\cdots\right), \\
& S_{34}=\frac{1}{8 \delta_{1}}\left(\Omega a_{144} A_{2} b_{12}-2 \Omega a_{334} A_{3} b_{12}-3 \Omega a_{333} A_{4} b_{12}+\cdots\right), \\
& s_{44}=\frac{1}{8 \delta_{2}}\left(\Omega a_{124} A_{2} b_{32}-2 \Omega a_{113} A_{2} b_{32}-\Omega a_{134} A_{3} b_{32}+\cdots\right), \text { and the other coefficients }
\end{aligned}
$$

have the same expressions as those given in equation (12).

The so-called frequency response equations are given by

$$
\begin{aligned}
& r_{1}^{2}\left(-\mu_{1}+s_{11} r_{1}^{2}+s_{12} r_{2}^{2}\right)^{2}+r_{1}^{2}\left(\frac{1}{3} \sigma_{2}-\rho_{1}-s_{31} r_{1}^{2}-s_{32} r_{2}^{2}\right)^{2}-\left(s_{14}^{2}+s_{34}^{2}\right) r_{2}^{2} r_{2}^{2}=0, \\
& r_{2}^{2}\left(-\mu_{2}+s_{21} r_{1}^{2}+s_{22} r_{2}^{2}\right)^{2}+r_{2}^{2}\left(\frac{1}{3} \sigma_{2}-\rho_{2}-s_{41} r_{1}^{2}-s_{42} r_{2}^{2}\right)^{2}-\left(s_{24}^{2}+s_{44}^{2}\right) r_{1}^{2} r_{2}^{2}=0 .
\end{aligned}
$$


Introducing the transformation $p_{21}=r_{1} \cos \gamma_{21}, q_{21}=r_{1} \sin \gamma_{21}, p_{22}=r_{2} \cos \gamma_{22}$, $q_{22}=r_{2} \sin \gamma_{22}$, into equation (15), and performing trigonometric manipulations leads to the following modulation equations in the Cartesian form:

$$
\begin{aligned}
\dot{p}_{21}= & -\mu_{1} p_{21}+\left(\rho_{1}-\frac{1}{3} \sigma_{2}\right) q_{21}+s_{11} p_{21}\left(p_{21}^{2}+q_{21}^{2}\right)+s_{12} p_{21}\left(p_{22}^{2}+q_{22}^{2}\right)+s_{31} q_{21}\left(p_{21}^{2}+q_{21}^{2}\right) \\
& +s_{32} q_{21}\left(p_{22}^{2}+q_{22}^{2}\right)+s_{14}\left(p_{22}^{2}-q_{22}^{2}\right)-2 s_{34} p_{22} q_{22}, \\
\dot{q}_{21}=- & \mu_{1} q_{21}-\left(\rho_{1}-\frac{1}{3} \sigma_{2}\right) p_{21}+s_{11} q_{21}\left(p_{21}^{2}+q_{21}^{2}\right)+s_{12} q_{21}\left(p_{22}^{2}+q_{22}^{2}\right)-s_{31} p_{21}\left(p_{21}^{2}+q_{21}^{2}\right) \\
& \quad-s_{32} p_{21}\left(p_{22}^{2}+q_{22}^{2}\right)-s_{34}\left(p_{22}^{2}-q_{22}^{2}\right)-2 s_{14} p_{22} q_{22}, \\
\dot{p}_{22}=- & \mu_{2} p_{22}+\left(\rho_{2}-\frac{1}{3} \sigma_{2}\right) q_{22}+s_{21} p_{22}\left(p_{21}^{2}+q_{21}^{2}\right)+s_{22} p_{22}\left(p_{22}^{2}+q_{22}^{2}\right)+s_{41} q_{22}\left(p_{21}^{2}+q_{21}^{2}\right) \\
& +s_{42} q_{22}\left(p_{22}^{2}+q_{22}^{2}\right)-s_{24}\left(p_{21} q_{22}-q_{21} q_{22}\right)-s_{44}\left(p_{21} q_{22}+p_{22} q_{21}\right), \\
\dot{q}_{22}=- & \mu_{2} q_{22}-\left(\rho_{2}-\frac{1}{3} \sigma_{2}\right) p_{22}+s_{21} q_{22}\left(p_{21}^{2}+q_{21}^{2}\right)+s_{22} q_{22}\left(p_{22}^{2}+q_{22}^{2}\right)-s_{41} p_{22}\left(p_{21}^{2}+q_{21}^{2}\right) \\
& -s_{42} p_{22}\left(p_{22}^{2}+q_{22}^{2}\right)-s_{24}\left(p_{22} q_{21}+p_{21} q_{22}\right)-s_{44}\left(p_{21} p_{22}-q_{21} q_{22}\right) .
\end{aligned}
$$

The eigenvalues of the corresponding Jacobian matrix of equation (17) determine the stability of the steady state solutions. The stability of the trivial solution is determined by the eigenvalues of the corresponding Jacobian matrix which is obtained by letting $p_{11}=q_{11}=p_{12}=q_{12}=0$. The four eigenvalues for the trivial solutions are given by $-\mu_{1} \pm i b_{2}$ and $-\mu_{2} \pm i b_{2}$, where $i$ is the imaginary unit, $b_{2}=\left|\rho_{2}-\frac{1}{3} \sigma_{2}\right|$. It is easy to note that the trivial solution is asymptotically stable if both $\mu_{1}>0$ and $\mu_{2}>0$.

Case III: $\Omega=2 \delta_{1}+\delta_{2}+\varepsilon \sigma_{3}$

In this case, the terms that produce secular or nearly secular terms are the trigonometric functions with the arguments of $\left(\delta_{1} t+\phi_{1}\right),\left(\delta_{2} t+\phi_{2}\right),\left(\Omega t-\delta_{1} t-\delta_{2} t-\phi_{1}-\phi_{2}\right)$ and $\left(\Omega t-2 \delta_{1} t-2 \phi_{1}\right)$ in the right hand sides of the equation, which is obtained by substituting solution (7) in equation (8). Eliminating these terms gives rise to the 
averaged equations that determine the amplitudes and phases of the free-oscillation terms for additive resonance Case III:

$$
\begin{aligned}
& \dot{r}_{1}=-\mu_{1} r_{1}+s_{11} r_{1}^{3}+s_{12} r_{1} r_{2}^{2}+s_{15} r_{1} r_{2} \cos \left(2 \gamma_{31}+\gamma_{32}\right)-s_{35} r_{1} r_{2} \sin \left(2 \gamma_{31}+\gamma_{32}\right), \\
& r_{1} \dot{\gamma}_{31}=\left(\frac{1}{3} \sigma_{3}-\rho_{1}\right) r_{1}-s_{31} r_{1}^{3}-s_{32} r_{1} r_{2}^{2}-s_{15} r_{1} r_{2} \sin \left(2 \gamma_{31}+\gamma_{32}\right)-s_{35} r_{1} r_{2} \cos \left(2 \gamma_{31}+\gamma_{32}\right), \\
& \dot{r}_{2}=-\mu_{2} r_{2}+s_{21} r_{1}^{2} r_{2}+s_{22} r_{2}^{3}+s_{25} r_{1}^{2} \cos \left(2 \gamma_{31}+\gamma_{32}\right)-s_{45} r_{1}^{2} \sin \left(2 \gamma_{31}+\gamma_{32}\right) \\
& r_{2} \dot{\gamma}_{32}=\left(\frac{1}{3} \sigma_{2}-\rho_{2}\right) r_{2}-s_{41} r_{1}^{2} r_{2}-s_{42} r_{2}^{3}-s_{25} r_{1}^{2} \sin \left(2 \gamma_{31}+\gamma_{22}\right)-s_{45} r_{1}^{2} \cos \left(2 \gamma_{31}+\gamma_{32}\right),
\end{aligned}
$$

where $\gamma_{31}=\frac{1}{3} \sigma_{3} T_{0}-\phi_{31}, \gamma_{32}=\frac{1}{3} \sigma_{3} T_{0}-\phi_{32}$,

$$
\begin{aligned}
& S_{15}=\frac{1}{8 \delta_{1}}\left(2 \Omega a_{133} A_{3} b_{12}-2 \Omega a_{114} A_{2} b_{12}-\Omega a_{123} A_{2} b_{12}+\cdots\right), \\
& S_{25}=\frac{1}{8 \delta_{2}}\left(\Omega a_{113} A_{3} b_{32}-2 \Omega a_{112} A_{2} b_{32}-\Omega a_{223} A_{3} b_{32}+\cdots\right), \\
& S_{35}=\frac{1}{8 \delta_{1}}\left(\Omega a_{124} A_{2} b_{12}-2 \Omega a_{113} A_{2} b_{12}-\Omega a_{134} A_{3} b_{12}+\cdots\right), \\
& S_{45}=\frac{1}{8 \delta_{2}}\left(\Omega a_{122} A_{2} b_{32}-\Omega a_{123} A_{3} b_{32}-\Omega a_{113} A_{4} b_{32}+\cdots\right), \text { and the other coefficients have }
\end{aligned}
$$

the same expressions as those given in equation (12).

The so-called frequency-response equations are then given by

$$
\begin{aligned}
& r_{1}^{2}\left(-\mu_{1}+s_{11} r_{1}^{2}+s_{12} r_{2}^{2}\right)^{2}+r_{1}^{2}\left(\frac{1}{3} \sigma_{3}-\rho_{1}-s_{31} r_{1}^{2}-s_{32} r_{2}^{2}\right)^{2}-\left(s_{15}^{2}+s_{35}^{2}\right) r_{1}^{2} r_{2}^{2}=0 \\
& r_{2}^{2}\left(-\mu_{2}+s_{21} r_{1}^{2}+s_{22} r_{2}^{2}\right)^{2}+r_{2}^{2}\left(\frac{1}{3} \sigma_{3}-\rho_{2}-s_{41} r_{1}^{2}-s_{42} r_{2}^{2}\right)^{2}-\left(s_{25}^{2}+s_{45}^{2}\right) r_{1}^{2} r_{1}^{2}=0
\end{aligned}
$$

Introducing the transformation $p_{31}=r_{1} \cos \gamma_{31}, q_{31}=r_{1} \sin \gamma_{31}, \quad p_{32}=r_{2} \cos \gamma_{32}$, $q_{32}=r_{2} \sin \gamma_{32}$, into equation (18), and performing algebraic manipulations leads to the following modulation equations in the Cartesian form:

$$
\begin{aligned}
\dot{p}_{31}=- & \mu_{1} p_{31}+\left(\rho_{1}-\frac{1}{3} \sigma_{3}\right) q_{31}+s_{11} p_{31}\left(p_{31}^{2}+q_{31}^{2}\right)+s_{12} p_{31}\left(p_{32}^{2}+q_{32}^{2}\right)+s_{31} q_{31}\left(p_{31}^{2}+q_{31}^{2}\right) \\
& +s_{32} q_{31}\left(p_{32}^{2}+q_{32}^{2}\right)+s_{15}\left(p_{31} p_{32}-q_{31} q_{32}\right)-s_{35}\left(p_{31} q_{32}+p_{32} q_{31}\right),
\end{aligned}
$$




$$
\begin{gathered}
\dot{q}_{31}=-\mu_{1} q_{31}-\left(\rho_{1}-\frac{1}{3} \sigma_{3}\right) p_{31}+s_{11} q_{31}\left(p_{31}^{2}+q_{31}^{2}\right)+s_{12} q_{31}\left(p_{32}^{2}+q_{32}^{2}\right)-s_{31} p_{31}\left(p_{31}^{2}+q_{31}^{2}\right) \\
\quad-s_{32} p_{31}\left(p_{32}^{2}+q_{32}^{2}\right)-s_{15}\left(p_{31} q_{32}-p_{32} q_{31}\right)-s_{35}\left(p_{31} p_{32}-q_{31} q_{32}\right) \\
\dot{p}_{32}=-\mu_{2} p_{32}+\left(\rho_{2}-\frac{1}{3} \sigma_{3}\right) q_{32}+s_{21} p_{32}\left(p_{31}^{2}+q_{31}^{2}\right)+s_{22} p_{32}\left(p_{32}^{2}+q_{32}^{2}\right)+s_{41} q_{32}\left(p_{31}^{2}+q_{31}^{2}\right) \\
\quad+s_{42} q_{32}\left(p_{32}^{2}+q_{32}^{2}\right)+s_{25}\left(p_{31}^{2}-q_{31}^{2}\right)-2 s_{45} p_{31} q_{31}, \\
\dot{q}_{32}=-\mu_{2} q_{32}-\left(\rho_{2}-\frac{1}{3} \sigma_{3}\right) p_{32}+s_{21} q_{32}\left(p_{31}^{2}+q_{31}^{2}\right)+s_{22} q_{32}\left(p_{32}^{2}+q_{32}^{2}\right)-s_{41} p_{32}\left(p_{31}^{2}+q_{31}^{2}\right) \\
\quad-s_{42} p_{32}\left(p_{32}^{2}+q_{32}^{2}\right)-s_{45}\left(p_{31}^{2}-q_{31}^{2}\right)-2 s_{25} p_{31} q_{31} .
\end{gathered}
$$

It is easy to notice that the stability of the trivial solution is determined by the following eigenvalues: $-\mu_{1} \pm i b_{3}$ and $-\mu_{2} \pm i b_{3}$, where $i$ is the imaginary unit, $b_{3}=\left|\rho_{2}-\frac{1}{3} \sigma_{3}\right|$. Therefore the trivial solution is asymptotically stable if both $\mu_{1}>0$ and $\mu_{2}>0$.

The averaged equations for three cases of additive resonances have been obtained analytically. In next section, the steady state solutions and their stability of the averaged equations will be studied by illustrative examples for a given set of the system parameters.

\section{Numerical Examples}

This section gives numerical results of the dynamic behaviour of additive resonance response of the system. As an illustrative example, consider a specific system with the system parameters in equation (3) given by $\mu=0.1, \omega=1.0, p=-0.4, q=-0.40219$, $\alpha=0.4, \beta=0.5, k_{1}=0.2, k_{4}=0.5, k_{2}=k_{3}=0.0$. It was found that an interaction of non-resonant Hopf bifurcations occurs when $\tau=5.46397$, where the frequencies of the two bifurcations are $\delta_{01}=1.28038$ and $\delta_{02}=0.71582$. It is easy to find from equations (9)-(11) that for this specific system, the combinational resonances of the additive type 
may appear in a certain interval of a small neighbourhood of the forcing frequencies at $\Omega_{0}=0.998096$ for Case I; $\Omega_{0}=2.71201$ for Case II; and $\Omega_{0}=3.27657$ for Case III.

When $\alpha_{1}=0.001, \alpha_{2}=-0.0053, e_{0}=0.12, \Omega_{0}=0.998$, the averaged equations for additive resonance Case I, which are obtained by substituting the numerical values of the corresponding coefficients in equation (12), are found to be

$$
\begin{aligned}
\dot{r}_{1}= & 0.02102 r_{1}-0.2335 r_{1}^{3}-0.0478 r_{1} r_{2}^{2}-0.016089 r_{2} \cos \left(\gamma_{11}+\gamma_{12}\right) \\
& +0.02052 r_{2} \sin \left(\gamma_{11}+\gamma_{12}\right), \\
r_{1} \dot{\gamma}_{11}= & \left(\frac{1}{2} \sigma_{1}-0.099652\right) r_{1}+0.5545 r_{1}^{3}+1.0091 r_{1} r_{2}^{2}+0.016089 r_{2} \sin \left(\gamma_{11}+\gamma_{12}\right) \\
& +0.02052 r_{2} \cos \left(\gamma_{11}+\gamma_{12}\right), \\
\dot{r}_{2}= & -0.0473715 r_{2}-1.5127 r_{1}^{2} r_{2}-0.6932 r_{2}^{3}+0.03968 r_{1} \cos \left(\gamma_{11}+\gamma_{12}\right) \\
& +0.022477 r_{1} \sin \left(\gamma_{11}+\gamma_{12}\right), \\
r_{2} \dot{\gamma}_{12}= & \left(\frac{1}{2} \sigma_{1}-0.059653\right) r_{2}+1.7226 r_{1}^{2} r_{2}+0.5886 r_{2}^{3}-0.03968 r_{1} \sin \left(\gamma_{11}+\gamma_{12}\right), \\
& +0.022477 r_{1} \cos \left(\gamma_{11}+\gamma_{12}\right) .
\end{aligned}
$$

The steady state solutions can be obtained by setting $\dot{r}_{1}, r_{1} \dot{\gamma}_{11}, \dot{r}_{2}$, and $r_{2} \dot{\gamma}_{12}$ in equation (21) equal to zero, and numerically solving the resulting algebraic equations. The stability of the solutions can be examined by finding the corresponding eigenvalues of the Jacobian matrix. Because two frequencies $\delta_{1}$ and $\delta_{2}$ are incommensurable, the steady state solutions of equation (21) correspond to quasi-periodic motions of the nonlinear system (3) under additive resonance Case I.

Figures 1(a) and (b) shows typical frequency-response curves for additive resonance Case I in the interval $\sigma_{1} \in[0.0483,0.06101]$. Stable solutions are represented by solid lines and unstable solutions by dashed lines. Solid and dashed lines will also be used to denote stable solutions and unstable solutions shown in frequency response curves for Cases II and III. The frequency response curve is an isolated closed curve in Figures 
1(a) and (b). The non-trivial solutions do not exist outside this closed curve. The closed curve consists of two branches. The upper branch of $r_{1}$ is stable with the four eigenvalues having negative real part. The lower branch of $r_{1}$ is unstable with the eigenvalues having positive real part. On the contrary, the upper branch of $r_{2}$ is unstable and the lower branch of $r_{2}$ is stable. The isolated two non-trivial solutions coexist with the trivial solution that is unstable in the interval $\sigma_{1}$.

As $\sigma_{1}$ increases from a small value, the amplitude $r_{1}$ of the free-oscillation term with natural frequency $\delta_{1}$ grows, whereas the amplitude $r_{2}$ of the free-oscillation term having natural frequency $\delta_{2}$ decreases. The amplitude $r_{1}$ is much higher than amplitude $r_{2}$, which means the motion corresponding to $\delta_{1}$ dominates while the motion relating to $\delta_{2}$ is smaller in amplitude but not negligible. Stable non-trivial solutions indicate that nonlinear response of the system under additive resonances comprises both the freeoscillation terms and forced terms as given in equation (7). Because the natural frequencies $\delta_{1}$ and $\delta_{2}$ are incommensurable, the system response under additive resonance Case I will exhibit quasi-periodic motion. Figures 1(c) and (d) shows the time history and phase portrait of quasi-periodic motion of the system for $\Omega_{0}=1.025$. A frequency spectral analysis has confirmed that the motion consists of three harmonic components, which are the natural frequencies $\delta_{1}$ and $\delta_{2}$, and the frequency of the excitation $\Omega$ satisfying the relationship (9). The numerical result on the existence of quasi-periodic motion is in good agreement with the analytical prediction.

Numerical simulations have also been performed for additive resonance Cases II and III. The values of system parameters and dummy parameters remained unchanged as those for Case I except the frequency of the excitation. Topologically equivalent response curves have been obtained for Case II. Figures 2(a) and (b) show frequency- 
response curves for Case II in the interval $\sigma_{2} \in[0.04924,0.0857233]$. Figure 2(c) and (d) show a quasi-periodic motion at $\Omega_{0}=2.712$. Figures 2(a) and (b) indicate that the motion relating to $\delta_{2}$ dominates while the motion corresponding to $\delta_{1}$ is smaller in amplitude. The quasi-periodic motion shown in Figure 2(c) is a quasi-periodic motion on a three-dimensional (3D) torus and contains three individual harmonic components: $\delta_{1}, \delta_{2}$ and $\Omega_{0}$. These three frequencies satisfy the additive resonance condition that is given by equation (10).

For additive resonance Case III, numerical simulation results are shown in Figure 3, where Figures 3(a) and (b) show frequency-response curves in the interval $\sigma_{3} \in[0.0758735,0.095176]$. Figure 3(c) and (d) show a quasi-periodic motion at $\Omega_{0}=3.278$. It is noted in Figures 3(a) and (b) that the amplitudes of two motions relating to $\delta_{1}$ and $\delta_{2}$ are comparable. The meeting points of stable and unstable branches were found to have saddle-node bifurcations. As the detuning $\sigma_{3}$ increases, the amplitude $r_{2}$ increases until it reaches the maximum and then decreases, whereas the amplitude $r_{1}$ increases until it meets the saddle-node point. This indicates that vibrational energy is transferred between these two motions via additive resonances. The quasi-periodic motion shown in Figure 3(c) involves three harmonic components: $\delta_{1}, \delta_{2}$ and $\Omega_{0}$, which satisfy the resonance condition given by equation (11).

When the combinational resonances of the additive types are not excited, the nonlinear response of the system was numerically found to exhibit periodic motion which consists of the forced terms only and has the forcing frequency. 


\section{Conclusion}

In a controlled van der Pol-Duffing oscillator without external excitation, periodic or quasi-periodic solutions may appear after the trivial equilibrium loses its stability via a non-resonant interaction of Hopf-Hopf bifurcations. Presence of a periodic external excitation in the controlled van der Pol-Duffing oscillator at non-resonant bifurcations of co-dimension two can induce three types of additive resonances as a result of interactions of the bifurcating solutions and the periodic excitation. Such interactions between the bifurcating solutions and the excitation could lead to very interesting phenomena. Particularly, when their natural frequencies are incommensurable, interactions may produce quasi-periodic motions.

The method of multiple scales has been used to study the steady state solutions of a system of coupled, weakly four-dimensional nonlinear differential equations, which represents the local flow on the centre manifold in the neighbourhood of non-resonant bifurcations of co-dimension two occurring in the controlled oscillator Three cases are considered for additive resonances: $2 \Omega=\delta_{1}+\delta_{2}+\varepsilon \sigma_{1} ; \quad \Omega=\delta_{1}+2 \delta_{2}+\varepsilon \sigma_{2}$; $\Omega=2 \delta_{1}+\delta_{2}+\varepsilon \sigma_{3}$

If the additive resonances are excited, the amplitudes of the free-oscillation terms admit three solutions; two non-trivial solutions and the trivial solution. Of two nontrivial solutions one is stable. The trivial solution is unstable. A stable non-trivial solution corresponds to a quasi-periodic motion of the original system. The quasiperiodic motion consists of three components having the frequency of the first Hopf bifurcation, the frequency of the second Hopf bifurcation, and the frequency of the excitation, respectively. The quasi-periodic motion is on a 3Dtorus, which can be viewed as a motion by adding a third periodic motion resulting from the periodic 
excitation to a quasi-period motion on a 2D torus having frequencies of two Hopf bifurcations. The three frequencies satisfy the additive resonance conditions.

The nonlinear system given by equation (1) is an infinite dimensional system in a mathematical sense. Under additive resonances this system has been found to exhibit quasi-periodic motions involving three frequencies. Except the forcing frequency, the other two frequencies are difficult to identify from the original system (1), as they do not relate to the natural frequency $\omega$, nor to its multiples. They came from two Hopf bifurcations occurring in the corresponding autonomous system. The observable behaviour of the non-autonomous system under additive resonances provides useful information for fault diagnosis. Presence of a complicated behaviour may indicate that the original system has undergone a certain bifurcation and induced certain types of additive resonances.

\section{References}

[1] Campbell, S.A., Belair, J., 1999 Resonant co-dimension two bifurcation in the harmonic oscillator with delayed forcing, Canadian Applied Mathematics Quarterly, vol.7, pp.218-238.

[2] Maccari, A., 2001 The response of a parametrically excited van der Pol oscillator to a time delay state feedback, Nonlinear Dynamics, vol.26, pp105-119.

[3] Xu, J., Chung, K.W., 2003 Effects of time delayed position feedback on a van der Pol-Duffing oscillator, Physica D, vol.180, pp.17-39.

[4] Xu, J., Yu, P., 2004, Delay-induced bifurcation in an nonautonomous system with delayed velocity feedbacks, International Journal of Bifurcation and Chaos, vol.14, pp.2777-2798. 
[5] Maccari, A., 2006 Vibration control for parametrically excited Lienard systems, International Journal of Non-Linear Mechanics, vol.41, pp.146-155.

[6] El-Bassiouny, A.F., 2006 Stability and oscillation of two coupled duffing equations with time delay state feedback, Physica Scripta, vol.74(6), pp.726-735.

[7] Das, J., Mallik, A.K., 2006 Control of friction driven oscillation by time-delayed state feedback, Journal of Sound and Vibration, vol.297(3-5), pp.578-594.

[8] Lazarevic, M.P., 2006 Finite time stability analysis of PD alpha fractional control of robotic time-delay systems, Mechanics Research Communications, vol.33(2), pp.269-279.

[9] Ji, J.C., Hansen, C.H., 2006 Stability and dynamics of a controlled van der PolDuffing oscillator, Chaos, Solitons and Fractals, vol.28(2), pp.555-570.

[10] Ji, J.C., 2006 Nonresonant Hopf bifurcations of a controlled van der Pol-Duffing oscillator, Journal of Sound and Vibration, vol.297(1-2), pp.183-199.

[11] Ji, J.C., Zhang, N., 2007 Nonlinear response of a forced van der Pol-Duffing oscillator at non-resonant bifurcations of codimension two, Chaos, Solitons \& Fractals (under review).

[12] Hale, J., Theory of Functional Differential Equations, Springer, New York, 1977.

[13] Hale, J.K., Verduyn Lunel, S.M., Introduction to Functional Differential Equations, Springer, New York, 1983.

[14] Halanay, A., Differential Equations, Stability, Oscillations, Time Lags, Academic Press, New York, 1966.

[15] Nayfeh, A.H., Mook, D.T., Nonlinear Oscillations, Wiley, New York, 1979. 


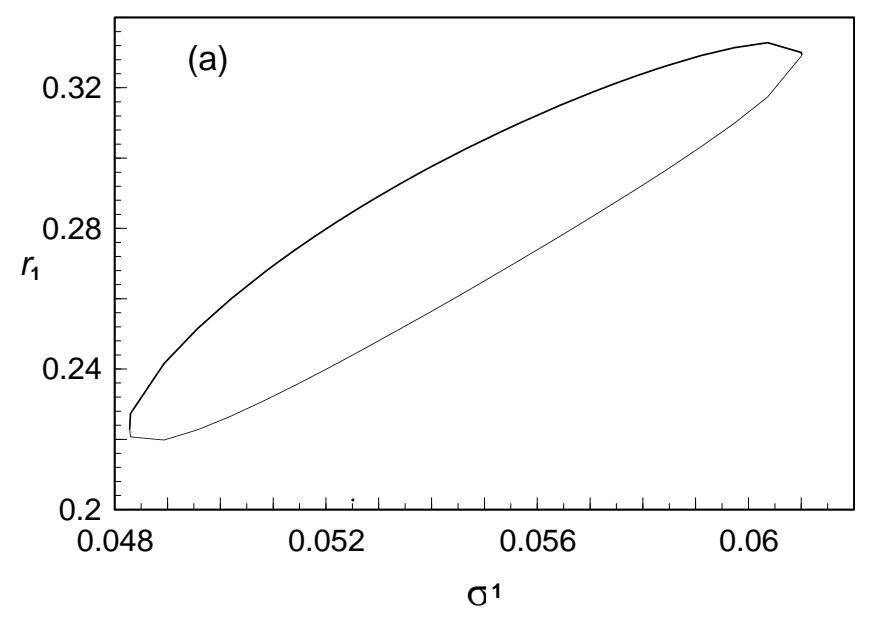

Figure 1(a)

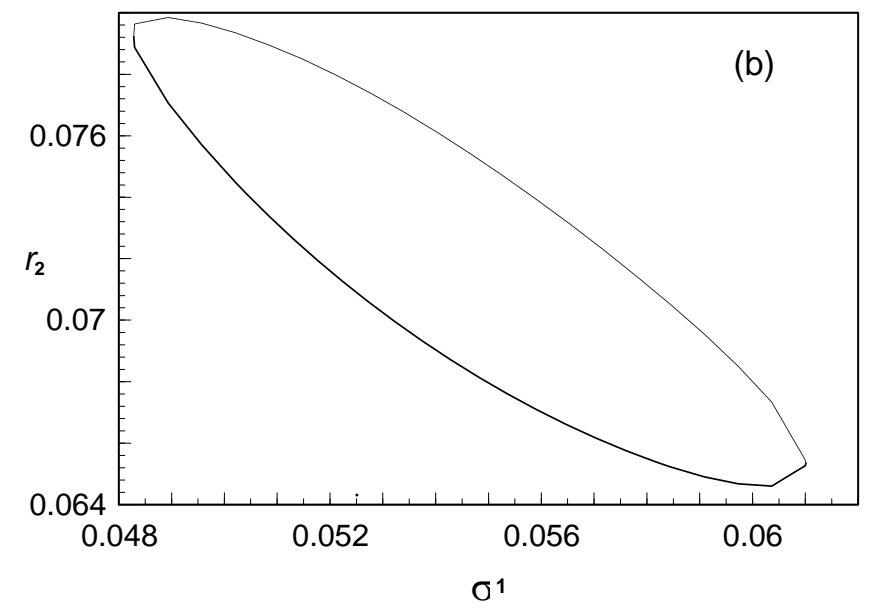

Figure 1(b)

Figure 1 Case I (continued) 


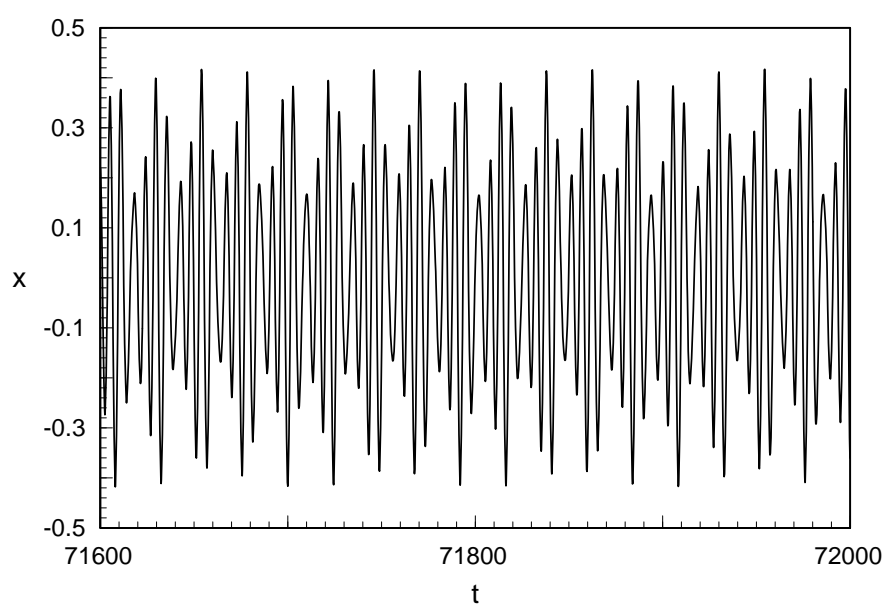

Figure 1(c )

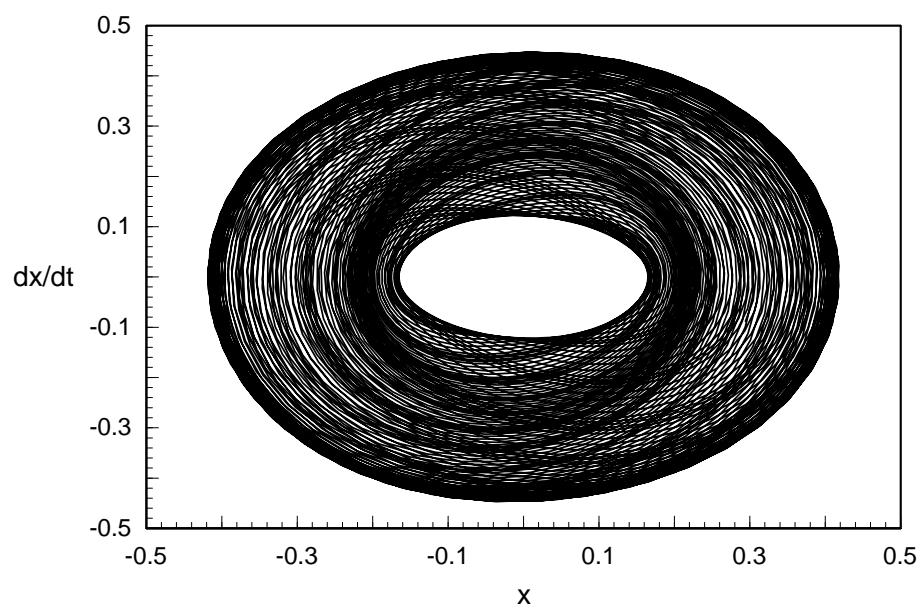

Figure 1(d)

Figure 1. Case I: (a) Frequency-response curve of $r_{1}$ for $\Omega_{0}=0.998$; (b) Frequencyresponse curve of $r_{2}$ for $\Omega_{0}=0.998$; (c) Time history of the quasi-periodic motion at $\Omega_{0}=1.025$; (d) Phase portrait of the quasi-periodic motion at $\Omega_{0}=1.025$. Solid lines in Figures (a) and (b) denote stable steady state solutions and dotted lines denote unstable solutions. 


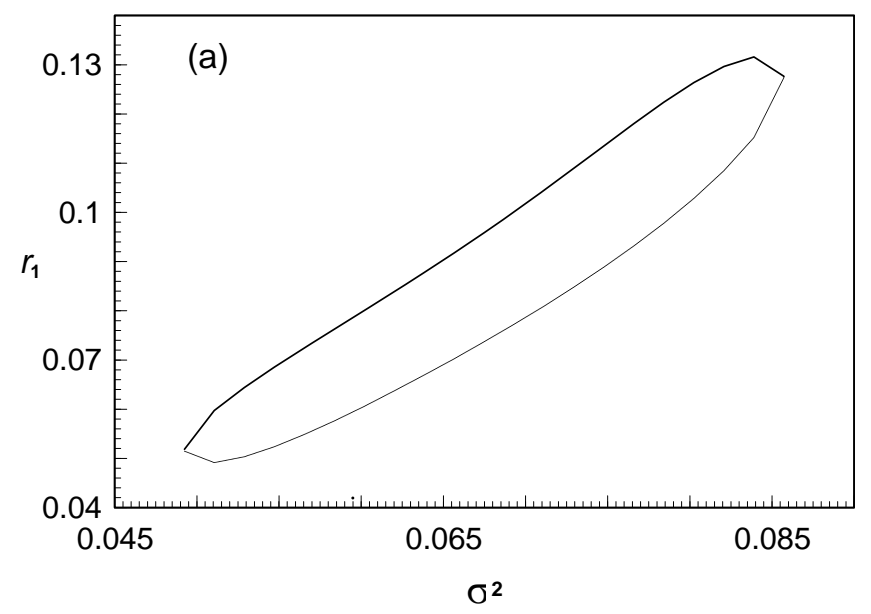

Figure 2(a)

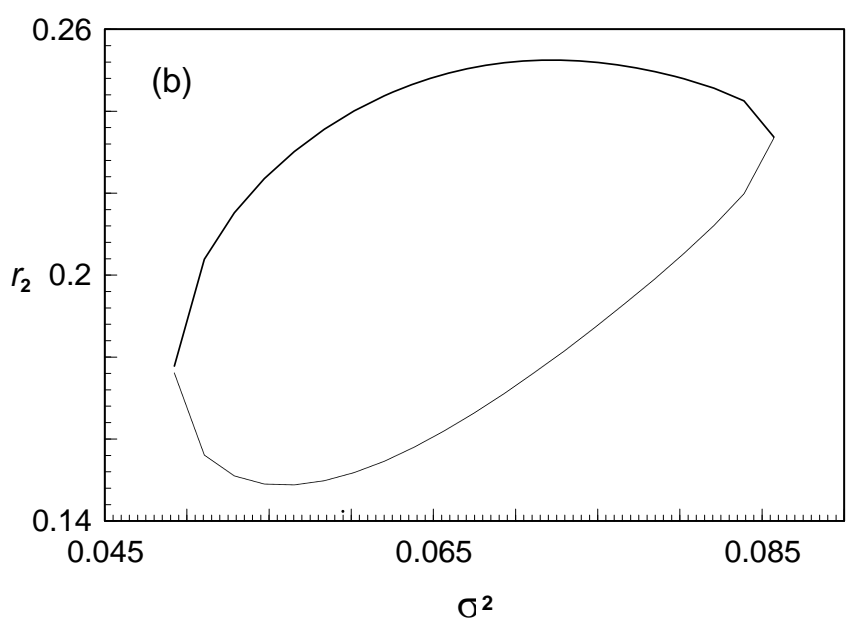

Figure 2(b)

Figure 2 Case II (continued) 


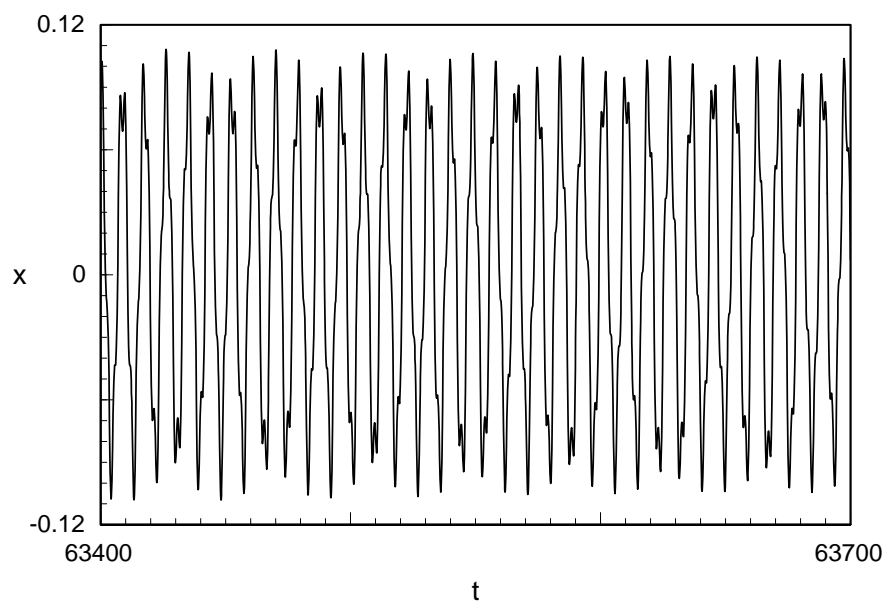

Figure 2(c )

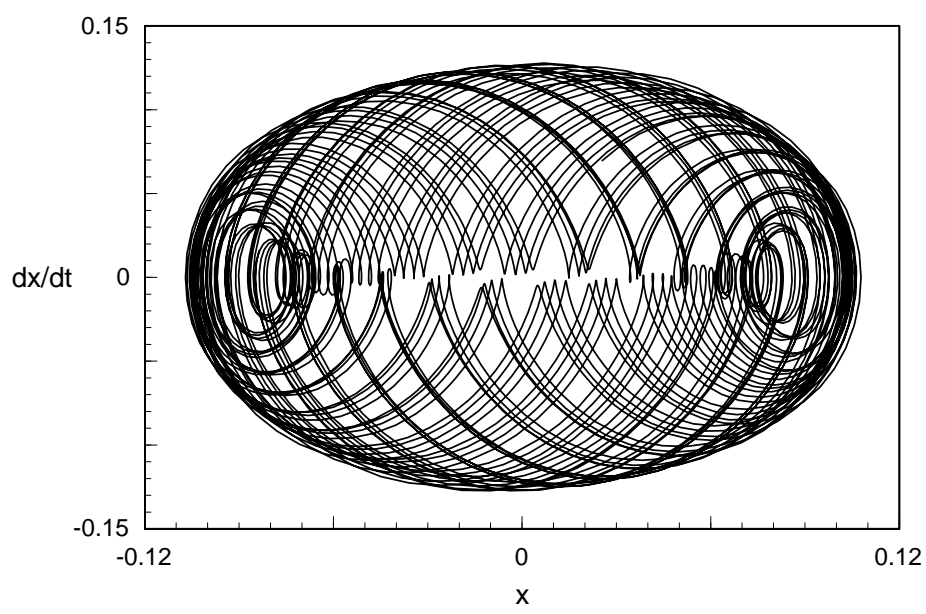

Figure 2(d)

Figure 2. Case II: (a) Frequency-response curve of $r_{1}$ for $\Omega_{0}=2.71$; (b) Frequencyresponse curve of $r_{2}$ for $\Omega_{0}=2.71$; (c) Time history of the quasi-periodic motion at $\Omega_{0}=2.712$; (d) Phase portrait of the quasi-periodic motion at $\Omega_{0}=2.712$. Solid lines in Figures (a) and (b) denote stable steady state solutions and dotted lines denote unstable solutions. 


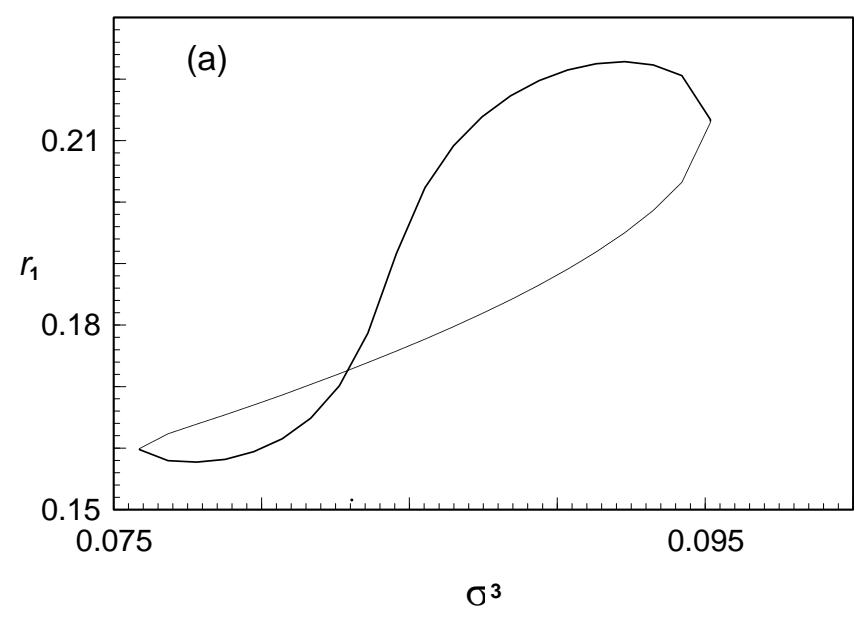

Figure 3(a)

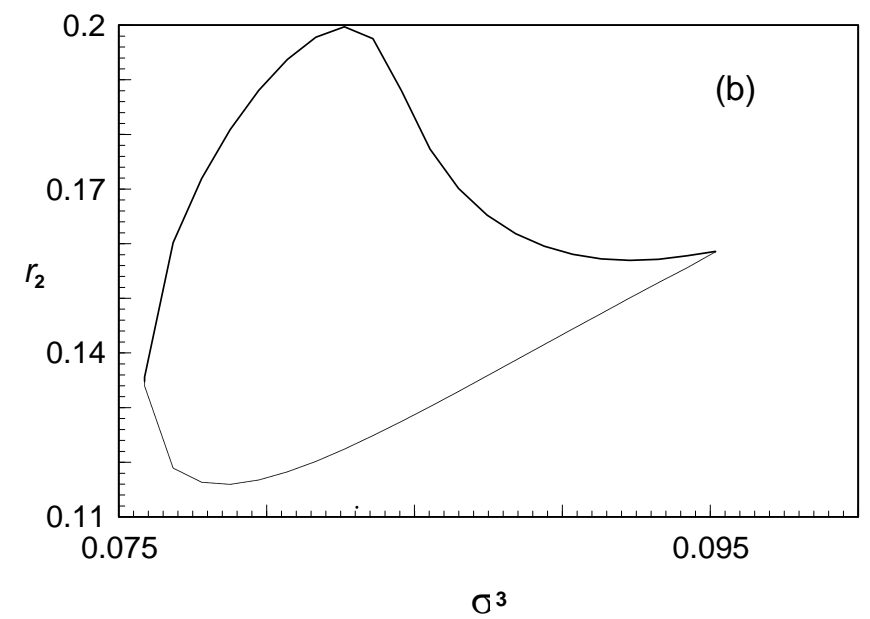

Figure 3(b)

Figure 3 Case III (continued) 


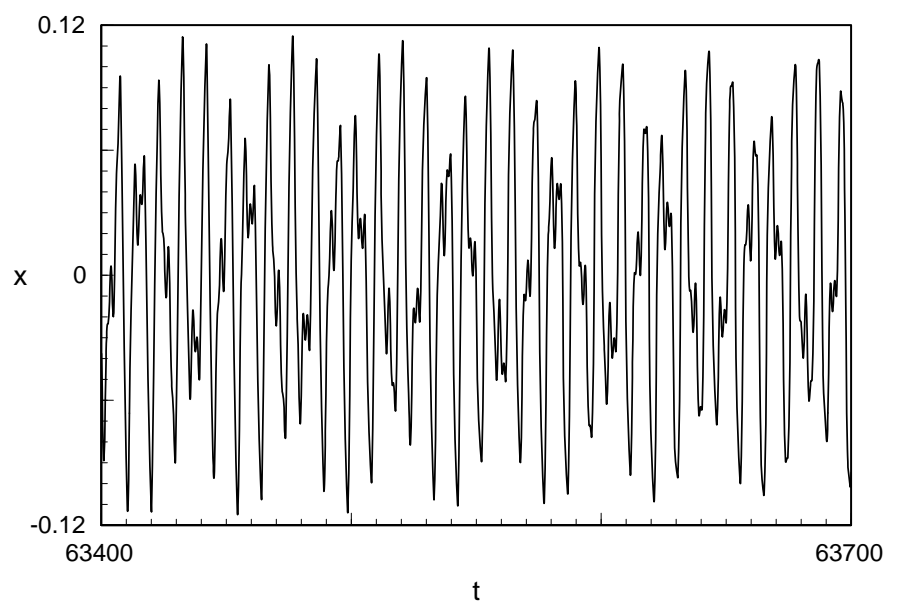

Figure 3(c )

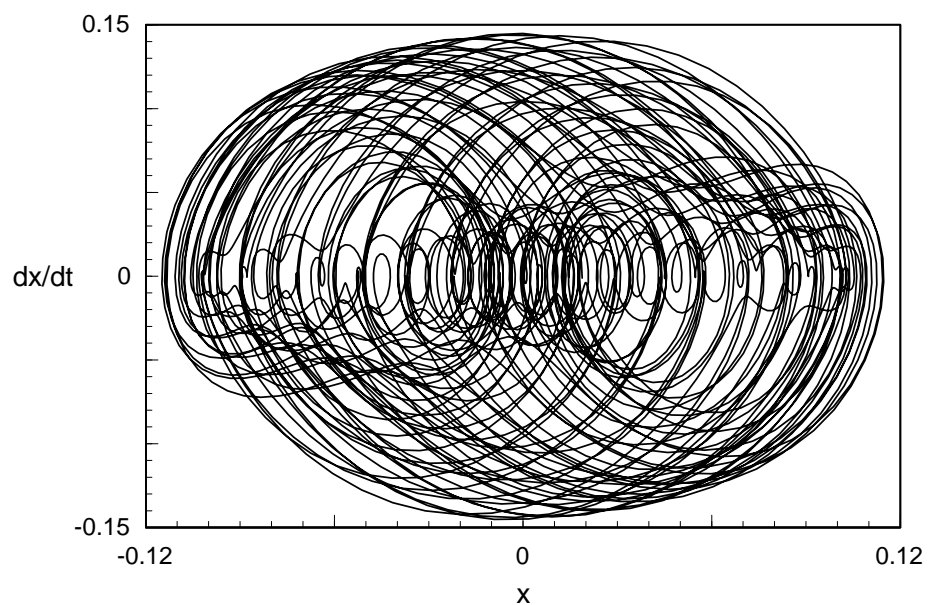

Figure 3(d)

Figure 3. Case III: (a) Frequency-response curve of $r_{1}$ for $\Omega_{0}=3.27$; (b) Frequencyresponse curve of $r_{2}$ for $\Omega_{0}=3.27$; (c) Time history of the quasi-periodic motion at $\Omega_{0}=3.278$; (d) Phase portrait of the quasi-periodic motion at $\Omega_{0}=3.278$. Solid lines in Figures (a) and (b) denote stable steady state solutions and dotted lines denote unstable solutions. 